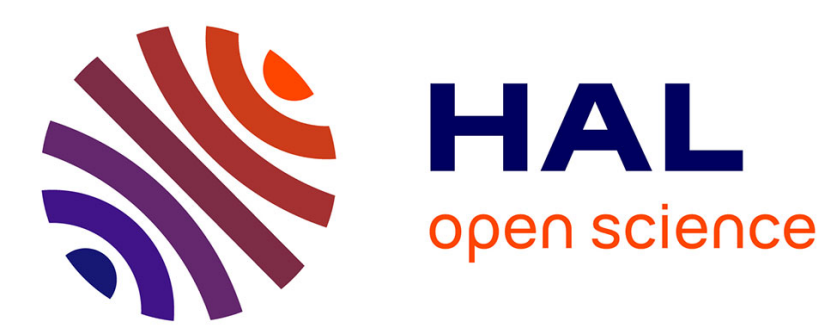

\title{
Les isolats de Phytophthora infestans (Mont.) de Bary sont-ils naturellement hétérozygotes?
}

\author{
Françoise Le Grand-Pernot, Fernande Dutfoy
}

\section{To cite this version:}

Françoise Le Grand-Pernot, Fernande Dutfoy. Les isolats de Phytophthora infestans (Mont.) de Bary sont-ils naturellement hétérozygotes?. Agronomie, 1986, 6 (10), pp.877-883. hal-00884834

\section{HAL Id: hal-00884834 \\ https://hal.science/hal-00884834}

Submitted on 1 Jan 1986

HAL is a multi-disciplinary open access archive for the deposit and dissemination of scientific research documents, whether they are published or not. The documents may come from teaching and research institutions in France or abroad, or from public or private research centers.
L'archive ouverte pluridisciplinaire HAL, est destinée au dépôt et à la diffusion de documents scientifiques de niveau recherche, publiés ou non, émanant des établissements d'enseignement et de recherche français ou étrangers, des laboratoires publics ou privés. 


\section{Les isolats de Phytophthora infestans (Mont.) de Bary sont-ils naturellement hétérozygotes?}

Françoise LE GRAND-PERNOT

avec la collaboration technique de Fernande DUTFOY

Laboratoire de Cryptogamie, associé au C.N.R.S. LA 86, Université Paris XI, Bâtiment 400, F 91405 Orsay hybride de plusieurs descendants.

Mots clés additionnels : Reproduction sexuelle, croissance à $10^{\circ} \mathrm{C}$ et $16^{\circ} \mathrm{C}$, sporulation, compatibilité sexuelle.

Additional key words : Sexual reproduction, growth at $10^{\circ} \mathrm{C}$ and $16{ }^{\circ} \mathrm{C}$, sporulation, mating type.

\section{INTRODUCTION}

De nos jours Phytophthora infestans (Mont.) de Bary, agent du mildiou de la pomme de terre représente encore un réel danger malgré la recherche de nouvelles variétés et de nouvelles molécules fongicides. Quels mécanismes génétiques peuvent rendre compte de l'aptitude du champignon à s'adapter aux moyens de protections que lui opposent sélectionneurs et chimistes?

Au Mexique, aire d'origine de $P$. infestans, le polymorphisme de l'espèce est assuré par la reproduction sexuelle, puisque les 2 sortes de thalle de type sexuel complémentaire $A_{1}$ et $A_{2}$ coexistent (GALLEGLY \& GALINDO, 1958). En Europe et en Amérique du Nord, bien que $P$. infestans ne soit présent que sous la forme $A_{1}$, il est possible par confrontation interspécifique d'induire réciproquement le cycle sexuel (SAVAGE et al., 1968 ; BOCCAS \& ZENTMYER, 1976 ; BOCCAS, 1978). Si les produits d'hybridation interspécifique avortent (BOCCAS \& ZENTMYER, 1976; BOCCAS,
1978), en revanche les produits autofécondés de chacune des espèces peuvent être nombreux et bien formés, leur existence résultant de l'hermaphrodisme potentiel des deux souches. Par conséquent l'hypothèse du rejet d'une phase sexuelle pour des isolats $A_{1}$ de $P$. infestans existant dans nos aires de culture ne semble pas actuellement fondée.

Cependant, montrer que des produits d'autofécondation sont susceptibles de se former n'a d'incidence pratique que s'ils peuvent être à l'origine de l'expression de nouvelles variations, c'est-à-dire si le mycélium est hétérocaryotique quel que soit le degré de ploïdie des noyaux, ou dans le cas d'une structure homocaryote si les noyaux sont diploïdes et hétérozygotes. Des travaux basés sur des données cytologiques et génétiques ont abouti chez les espèces de Phytophthora hétérothalliques à des résultats contradictoires en suggérant l'hypothèse haploïde (LAviola \& GALLEGLY, 1969 ; GALLEGLY, 1970 ; TIMMER et al., 1970 ; LAVIOLA, 1974) ou diplö̈de (SATOUR \& BUTLER, 1968 : SHAW \& KHAKI, 1971 ; BOCCAS, 1971 ; 
SHAW et al., 1973 ; KHAKI \& SHAW, 1974). Par d'autres approches nous avons pu au laboratoire déterminer clairement la nature diploïde du cycle de développement de plusieurs Phytophthora (LE Grand-PERnot \& Pellegrin, 1976) dont $P$. infestans (PAILlARD, 1977).

Ainsi l'assurance d'un cycle biologique diploïde permet d'envisager une structure hétérozygote partielle ou totale chez les isolats naturels qui serait une source importante de variabilité.

Pour tenter de répondre à cette hypothèse nous avons tout d'abord analysé à travers une descendance intraspécifique de première génération, la transmission de caractères aisément observables comme la croissance et la morphologie des thalles à $10^{\circ} \mathrm{C}$, la vitesse de croissance et la sporulation des souches cultivées à $16{ }^{\circ} \mathrm{C}$ (température généralement retenue pour faire croître ce champignon au laboratoire).

\section{MATÉRIEL ET MÉTHODES}

\section{A. Les souches}

Quatre souches parentales de Phytophthora infestans (A, D, St et $\mathrm{Cv}$ ) d'origine géographique différente, et 57 descendants de $1^{\text {re }}$ génération issus des différentes confrontations possibles sont entretenus par bouturage sur milieu $C$ gélosé (LE GRANDPERNOT, 1981).

Parmi les 57 descendants obtenus sur des dizaines de milliers d'oospores isolées, 50 proviennent du croisement A par St, 5 de l'association A avec D, enfin 2 seulement sont originaires de la confrontation entre $\mathrm{A}$ et $\mathrm{Cv}$.

Périodiquement, tous les 2 mois environ, les souches parentales et leurs descendants sont inoculés à des tranches de tubercule de pomme de terre Bintje, afin d'entretenir leur agressivité.
Les diverses caractéristiques des souches parentales vis-à-vis des composantes étudiées sont résumées dans le tableau 1.

\section{B. Morphologie des thalles et degré d'envahissement après 30 jours de culture à $10^{\circ} \mathrm{C}$}

Un lot de souches dont la croissance a été initiée à $16^{\circ} \mathrm{C}$ est transféré dans une étuve à $10^{\circ} \mathrm{C}$, tandis que l'autre lot reste durant toute l'expérience à $16^{\circ} \mathrm{C}$. Au bout de 30 jours toutes les cultures sont observées. En comparant les deux séries entre elles, on note l'influence que peut avoir un séjour prolongé à $10^{\circ} \mathrm{C}$ sur la morphologie du thalle, et la vitesse de propagation des hyphes sur le milieu nutritif gélosé.

\section{Elongation radiale moyenne par jour pour les souches cultivées à $16^{\circ} \mathrm{C}$}

Pour chacune des souches 14 boutures, calibrées à l'emporte-pièce de $9 \mathrm{~mm}$ de diamètre et prélevées sur des thalles âgés de 4 jours, sont déposées au centre des boîtes de Pétri contenant du milieu $\mathrm{C}$ gélosé additionné d'une solution d'antibiotiques (100 p.p.m. de Pimafucine, 50 p.p.m. de Polymixine et 50 p.p.m. de Penicilline). Tous les deux jours à partir du $3^{\mathrm{e}}$ jour de mise en culture à $16^{\circ} \mathrm{C}$, on mesure le diamètre des thalles. Pour chacune des 61 courbes de croissance, nous avons testé la linéarité de la régression entre 4 et 8 jours de croissance à l'aide d'un modèle fondé sur la régression non linéaire (DAGNELIE, 1970). L'élongation journalière moyenne a été estimée par le calcul du coefficient de régression, dont on a précisé les limites (DAGNELIE, 1970).

\section{Evaluation de la quantité moyenne de sporocystes formés par thalles à $16^{\circ} \mathrm{C}$}

Pour chaque souche, la surface de 3 thalles âgés de 13 jours est recouverte par $10 \mathrm{ml}$ d'eau stérile, puis

TABLEAU 1

Quelques caractéristiques des souches parentales sur milieu $C$. Some characteristics of parental strains on $C$ medium.

\begin{tabular}{|c|c|c|c|c|}
\hline \multirow[b]{3}{*}{ Souche } & \multicolumn{4}{|c|}{ Composantes étudiées } \\
\hline & \multicolumn{2}{|c|}{$\begin{array}{l}\text { Culture à } 10^{\circ} \mathrm{C} \\
\text { pendant } 30 \text { jours }\end{array}$} & \multicolumn{2}{|c|}{$\begin{array}{l}\text { Culture à } 16^{\circ} \mathrm{C} \\
\text { pendant } 13 \text { jours }\end{array}$} \\
\hline & $\begin{array}{l}\% \text { surface } \\
\text { couverte } \\
\text { par le thalle }\end{array}$ & $\begin{array}{c}\text { faciès } \\
\text { du thalle }\end{array}$ & $\begin{array}{c}\text { vitesse } \\
\text { de croissance } \\
\mathrm{mm} / \mathrm{j}\end{array}$ & $\begin{array}{c}\text { nombre moyen } \\
\text { de sporocystes } \\
\text { par thalle }\end{array}$ \\
\hline$\frac{\mathrm{A}}{\text { (type } \mathrm{A}_{2} \text { ) }}$ & 0 & & $3,84 \pm 0,2$ & $7,2.10^{3}$ \\
\hline $\begin{array}{c}\mathrm{D} \\
\text { (type } A_{1} \text { ) }\end{array}$ & 100 & $\begin{array}{l}\text { mycélium ras } \\
\text { au centre } \\
\text { épais aux bords }\end{array}$ & $4,15 \pm 0,12$ & $1,5.10^{7}$ \\
\hline $\begin{array}{c}\mathrm{St} \\
\text { (type } \mathrm{A}_{1} \text { ) }\end{array}$ & 100 & $\begin{array}{l}\text { mycélium ras, } \\
\text { régulièrement } \\
\text { moucheté }\end{array}$ & $4,51 \pm 0,11$ & $1,4 \cdot 10^{7}$ \\
\hline $\begin{array}{c}\mathrm{Cv} \\
\text { (type } \mathrm{A}_{1} \text { ) }\end{array}$ & 45 & $\begin{array}{l}\text { mycélium aérien } \\
\text { abondant } \\
\text { cotonneux }\end{array}$ & $4,07 \pm 0,13$ & $9.10^{6}$ \\
\hline
\end{tabular}


grattée à l'aide d'une spatule en verre pour en détacher les sporocystes.

Chacune de ces trois suspensions est estimée 2 fois par comptage à la cellule Malassez. Afin de déterminer si tous les échantillons proviennent d'une même population, et si les fluctuations observées sont dues au hasard, un test $X^{2}$ a été effectué. Ce n'est qu'après cette vérification que nous avons calculé une concentration moyenne de sporocystes par thalle.

\section{RÉSULTATS}

\section{A. Morphologie et degré d'envahissement des thalles après 30 jours de culture à $10^{\circ} \mathrm{C}$}

Les 4 souches parentales ( $\mathrm{A}, \mathrm{D}, \mathrm{St}$ et $\mathrm{Cv}$ ) ont à $16{ }^{\circ} \mathrm{C}$ une bonne vitesse de croissance (tabl. 1), proche (cas de $\mathrm{A}, \mathrm{D}$ et $\mathrm{Cv}$ ) ou supérieure (cas de $\mathrm{St}$ ) à celle de $4 \mathrm{~mm} / \mathrm{j}$ signalée par WATERHOUSE (1963) comme optimum pour l'espèce Phytophthora infestans.

Les hyphes des souches $\mathrm{D}$ et St initiées à $16^{\circ} \mathrm{C}$ puis transférées dans une enceinte à $10^{\circ} \mathrm{C}$ poursuivent leur développement et forment un thalle qui envahit en 2 à 3 semaines toute la boîte de Pétri ; celles de la souche $\mathrm{Cv}$ croissent très lentement puisqu'après 1 mois de culture à $10{ }^{\circ} \mathrm{C}$ elles n'occupent que $45 \%$ de la surface totale de la boîte. Enfin les hyphes de la souche $\mathrm{A}$, toujours initiées à $16^{\circ} \mathrm{C}$, n'évoluent pas après leur transfert à $10^{\circ} \mathrm{C}$; au bout de 30 jours les quelques rares filaments grêles observés sur ces boîtes correspondent à ceux formés lors du bref passage à $16{ }^{\circ} \mathrm{C}$.

Parmi 47 descendants du croisement A par St testés à $10^{\circ} \mathrm{C}$ (tabl. 2A) 38 se sont comportés comme le parent $\mathrm{St}$ : ils poursuivent leur croissance à cette température et occupent toute la surface de la boîte de Pétri au moment de la lecture ; 2 descendants ont réagi strictement comme le parent $\mathrm{A}$, en interrompant leur croissance dès leur transfert à $10{ }^{\circ} \mathrm{C}$; enfin 7 se sont révélés des phénotypes intermédiaires : la croissance du thalle n'est ni nulle, comme pour A, ni rapide comme pour St, le mycélium est plus ou moins envahissant, le degré de couverture de la boîte de Pétri après un mois de culture à $10^{\circ} \mathrm{C}$ varie d'un descendant à l'autre.

Dans ce même tableau 2B, on peut remarquer que les 5 descendants de la confrontation A par D conduisent à des résultats voisins des précédents, puisque 3 d'entre eux se comportent comme le parent $D, 1$ comme le parent $\mathrm{A}$, et que le dernier est intermédiaire. Un seul descendant dans le $3^{e}$ type de confrontation a pu être testé (tabl. 2C), il se distingue de A et de Cv en manifestant au bout de 30 jours une élongation bien supérieure à celle du parent $\mathrm{Cv}$.

Aux diverses aptitudes des thalles à croître à $10^{\circ} \mathrm{C}$ s'ajoutent les différents morphotypes observés à cette même température.

Les souches parentales $\mathrm{A}, \mathrm{D}$ et $\mathrm{Cr}$ cultivées à $16^{\circ} \mathrm{C}$ ont une morphologie voisine, leurs thalles possèdent 1 mycélium aérien abondant et cotonneux. A cette même température la souche $\mathrm{St}$ se distingue des 3 autres par un réseau mycélien moins aérien et plus épars. Transférés en début de croissance à $10^{\circ} \mathrm{C}$, les 4 souches parentales $\mathrm{A}, \mathrm{D}$, St et $\mathrm{Cv}$ vont évoluer différemment au cours de cette période et conduire à 4 phénotypes culturaux distincts (tabl. 1).

Les thalles de la souche St se caractérisent par un mycélium aérien, peu dense très ras, finement et régulièrement moucheté par des petits pelotons d'hyphes ; ceux de la souche $\mathrm{D}$ ont à $10{ }^{\circ} \mathrm{C}$ moins de filaments aériens au centre et prennent un aspect plutôt ras, en périphérie, le mycélium aérien est abondant et forme une couronne duveteuse. Les thalles de la souche $\mathrm{Cv}$ conservent la même morphologie qu'à $16^{\circ} \mathrm{C}$ : un mycélium aérien abondant et cotonneux ; enfin rappelons que la souche $\mathrm{A}$ ne croît pas à $10^{\circ} \mathrm{C}$.

Les descendants du croisement A par St se répartissent en plusieurs morphotypes (tabl. 2A) : le premier $(\mathrm{Mla}, \mathrm{b}, \mathrm{c})$ ressemble au faciès de la souche St cultivée à $10^{\circ} \mathrm{C}(\mathrm{M} 1 \mathrm{~b})$ certains descendants présentent moins de petits agrégats (M1a), d'autres en possèdent beaucoup plus (M1c), pour ces derniers il existe une convergence morphologique entre eux et les thalles du parent St cultivés à $16^{\circ} \mathrm{C}$. Le deuxième $(\mathrm{M} 2 \mathrm{a}, \mathrm{b})$ correspond aux descendants ayant soit exactement le même comportement que la souche $\mathrm{A}$ après son transfert à $10^{\circ} \mathrm{C}$ (M2a), soit le phénotype des thalles de cette même souche développés à $16^{\circ} \mathrm{C}$. Enfin le troisième réunit tous les cas dont le seul point commun est de se distinguer morphologiquement des souches $\mathrm{A}$ et St cultivées aux deux températures considérées $\left(10{ }^{\circ} \mathrm{C}\right.$ et $\left.16{ }^{\circ} \mathrm{C}\right)$.

Les produits des confrontations A par D ne sont pas non plus tous identiques (tabl. 2B). Une même morphologie des thalles $\mathrm{Cv}$ cultivés à $10^{\circ} \mathrm{C}$ et $16^{\circ} \mathrm{C}$ et des thalles $\mathrm{A}$ à $16^{\circ} \mathrm{C}$ nous empêche de connaître l'origine de l'unique descendant analysé (tabl. 2C).

Quel que soit le caractère examiné dans l'analyse du comportement des descendants à $10^{\circ} \mathrm{C}$, on observe une diversité des phénotypes, certains s'identifient à ceux des parents, les autres ne peuvent pas être assimilés à une population unique. Ces remarques s'appliquent aussi lorsque l'on considère simultanément ces deux composantes. Dans certains cas, il semble possible d'attribuer une origine autofécondée ou hybride de certains descendants. Lorsque des zygotes réassocient les caractères parentaux de $\mathrm{A}$ et de St $(\mathrm{I} / \mathrm{Mlb}-\mathrm{I} / \mathrm{Mlc}$ I/M3 - St/M2b - St/M3), ils pourrraient résulter d'une hybridation entre ces deux souches. Quand ils se comportent à $10{ }^{\circ} \mathrm{C}$ comme le parent $\mathrm{A}$ (A/M2a) ou strictement comme le parent St à la même température (St/Mlb), une origine autofécondée peut être soupçonnée.

Les quelques descendants de la confrontation $\mathrm{A}$ et D seraient aussi d'origine différente : autofécondations de $A$ pour w65 et de $D$ pour w51, et hybridations entre $\mathrm{A}$ et $\mathrm{D}$ pour les 3 autres zygotes.

\section{B. Etude du signe de compatibilité sexuelle}

En confrontant chacun des descendants avec 2 souches de référence $A_{1}$ et $A_{2}$ on peut déterminer son type de compatibilité sexuelle : un descendant est qualifié de $\mathrm{A}_{2}$ lorsque sa confrontation est fructueuse avec une souche $A_{1}$ et stérile avec une souche $A_{2}$; un autre sera de compatibilité $A_{1}$ si l'initiation de la reproduction sexuelle s'effectue uniquement en pré- 
TABLEAU 2

Phénotype des thalles et pourcentage de surface couverte par ces thalles après 30 jours de culture à $10{ }^{\circ} \mathrm{C}$. Phenotype of thallus and percentage of surface covered by thalli after 30 days growth at $10^{\circ} \mathrm{C}$.

A : confrontation A par St

\begin{tabular}{|c|c|c|c|c|c|c|c|}
\hline & \multicolumn{7}{|c|}{ Morphotypes } \\
\hline & & M1 & & M2 & & M3 & \\
\hline & Mla & M1b & Mlc & M2a & $\mathrm{M} 2 \mathrm{~b}$ & & \\
\hline $\begin{array}{c}\mathrm{A} \\
(0 \%)\end{array}$ & & & & $\begin{array}{c}\text { type } \\
\text { parental A } \\
\text { w28* } \\
\text { w37 }\end{array}$ & & & $\epsilon: 2$ \\
\hline$(16$ à $79 \%)$ & & $\begin{array}{ll}w 17 & (79 \%) \\
w 20 & (79 \%) \\
w 21^{*} & (20 \%) \\
w 22^{*} & (20 \%)\end{array}$ & $w 52 *(52 \%)$ & & & $\begin{array}{l}\text { w31 }(52 \%) \\
\text { w18 }(16 \%)\end{array}$ & $\epsilon: 7$ \\
\hline $\begin{array}{c}\text { St } \\
(100 \%)\end{array}$ & $\begin{array}{l}\text { w14* }-w 49^{*} \\
\text { w } 58^{* *}\end{array}$ & $\begin{array}{c}\text { type } \\
\text { parental St } \\
\text { w1 -w2-w3 } \\
\text { w4 -w5-w6 } \\
\text { w7 -w } 8-w 11 \\
\text { w46-w48 } \\
\text { w64 }\end{array}$ & $\begin{array}{l}w 29-w 41^{* *} \\
w 50^{* *}-w 59^{*} \\
w 61^{* *}-w 63\end{array}$ & & $\begin{array}{ll}w 10 & -w 16^{* *} \\
w 19 & -w 23 \\
w 24^{*} & -w 27 \\
w 32 & -w 33 \\
w 34^{* *}-w 39 \\
w 42 & -w 47^{* *} \\
w 55^{*} & -w 62^{*}\end{array}$ & $\begin{array}{l}\text { w } 15-w 35 * * \\
\text { w60 }\end{array}$ & $\epsilon: 38$ \\
\hline
\end{tabular}

M1 : morphotype ayant au moins une origine St.

Mla : mycélium moins dense que St à $10^{\circ} \mathrm{C}$.

MIb : mycélium comme St à $10^{\circ} \mathrm{C}$.

Mlc : mycélium plus dense que St à $10^{\circ} \mathrm{C}$ (voisin de St à $16^{\circ} \mathrm{C}$ ).

M2 : morphotype ayant au moins une origine $\mathrm{A}$.

M2a : comme A à $10^{\circ} \mathrm{C}$.

$\mathrm{M} 2 \mathrm{~b}$ : voisin ou identique à $\mathrm{A} 16^{\circ} \mathrm{C}$

M3 : morphotype différent de $\mathrm{A}$ et de St à $10{ }^{\circ} \mathrm{C}$ et $16^{\circ} \mathrm{C}$.

Type de compatibilité sexuelle : $w . . * \mathrm{~A}_{1} / / \mathrm{A}_{2}$

$$
\begin{array}{ll}
w_{1 *}^{*} & \mathrm{~A}_{2} \\
\mathrm{w}_{2} & \mathrm{~A}_{1}
\end{array}
$$

B : Confrontation A par D

\begin{tabular}{|c|c|c|c|c|c|}
\hline \multicolumn{6}{|c|}{ Morphotypes } \\
\hline \multirow[b]{2}{*}{$\stackrel{A}{A}$} & M2a & comme D à $10^{\circ} \mathrm{C}$ & $\begin{array}{l}\text { comme } \mathrm{A} \text { ou } \mathrm{D} \\
\text { à } 16^{\circ} \mathrm{C}\end{array}$ & $\begin{array}{l}\text { différents de } \mathrm{A} \text { et } \mathrm{D} \\
\text { à } 10^{\circ} \mathrm{C} \text { et } 16^{\circ} \mathrm{C}\end{array}$ & \\
\hline & $\begin{array}{l}\text { type parental } \mathrm{A} \\
\text { w65 }\end{array}$ & & & & $\epsilon: 1$ \\
\hline$\stackrel{I}{I}$ & & \multicolumn{3}{|c|}{ w66 $(66 \%)$} & $\epsilon: 1$ \\
\hline \multirow[t]{4}{*}{$\begin{array}{c}D \\
(100 \%)\end{array}$} & w5 I & type parental $\mathrm{D}$ & $w 44 *$ & w67 & $\epsilon: 3$ \\
\hline & \multicolumn{5}{|c|}{$\mathrm{C}$ : Confrontation A par $\mathrm{Cv}$} \\
\hline & \multicolumn{5}{|c|}{ Morphotypes } \\
\hline & M2a & & $\begin{array}{l}\text { comme } \mathrm{A} \text { à } 16^{\circ} \mathrm{C} \text { ou } \\
\mathrm{C} v \text { à } 10^{\circ} \mathrm{C} \text { et } 16^{\circ} \mathrm{C}\end{array}$ & $\begin{array}{l}\text { différents de } \mathrm{A} \text { et } \mathrm{CV} \\
\text { à } 10^{\circ} \mathrm{C} \text { et } 16^{\circ} \mathrm{C}\end{array}$ & \\
\hline $\begin{array}{c}\mathrm{A} \\
(0 \%)\end{array}$ & \multicolumn{5}{|l|}{ type parental A } \\
\hline $\begin{array}{c}C V \\
(45 \%)\end{array}$ & \multicolumn{5}{|c|}{ type parental $\mathrm{Cv}$} \\
\hline$>\underset{(80 \%)}{A}>\mathrm{C}$ & \multicolumn{5}{|c|}{ w69 $(80 \%)$} \\
\hline
\end{tabular}


sence de la souche parentale $A$. Enfin on parlera de thalle ambivalent $\left(\mathrm{A}_{1} / / \mathrm{A}_{2}\right)$ quand les formations sexuelles apparaitront pour toute association contractée avec $A_{1}$ ou $A_{2}$ (LE GRAND-PERNOT, 1983). Les résultats sont présentés dans les tableaux $2 \mathrm{~A}, 2 \mathrm{~B}, 2 \mathrm{C}$ de la façon suivante : lorsque le nom du descendant possède 1 astérisque il est ambivalent $A_{1} / / A_{2}$, lorsqu'il en a 2 il est de type $A_{2}$ enfin lorsqu'il n'a rien, il est de type $A_{1}$.

Les travaux de MORTIMER et al. (1977) ceux de MAÏA (1986) nous renseignent sur l'origine éventuelle des descendants. Ainsi, les descendants ambivalents $A_{1} / / A_{2}$ peuvent résulter de l'autofécondation de la souche $\mathrm{A}_{2}$ ou de l'hybridation entre les deux partenaires. L'autofécondation d'une souche de type $\mathrm{A}_{1}$ conduit à une descendance homogène $A_{1}$, en revanche celle d'une souche $A_{2}$ donne naissance à une descendance variée de type $A_{1}, A_{2}$ ou $A_{1} / / A_{2}$.

Le fait que tous les descendants ressemblant strictement à la souche St (St/M1b, tabl. 2A) expriment dès leur formation le signe de compatibilité $\mathrm{A}_{1}$ renforce l'idée déjà exprimée précédemment de leur origine autofécondée.

L'ambivalence ou le type $A_{2}$ des souches de morphotype M1a et M1c suppose que ces descendants ont une origine hybride.

\section{Elongation radiale moyenne par jour des souches parentales et de leurs descendants cultivés à $16^{\circ} \mathrm{C}$}

Le comportement des descendants du croisement A par St est très variable, la croissance peut être soit égale à l'un des parents, soit inférieure ou supérieure aux 2 parents soit enfin intermédiaire. Mais il n'existe pas de classes bien séparées. Le passage d'une valeur à une autre s'effectue graduellement, lorsque l'on classe les souches par ordre croissant d'élongation radiale moyenne journalière (fig. 1). La dispersion de ces valeurs, et particulièrement celles obtenues chez les autofécondés de la souche $\mathrm{St}(\mathrm{s})$, suggère que ce caractère est probablement de nature quantitative et qu'il est gouverné par plusieurs gènes. Les quelques descendants obtenus en confrontant A par D ont une vitesse de croissance inférieure ou très inférieure à celle des 2 parents. Malgré le nombre réduit de représentants, on observe de nouveau une dispersion des valeurs. Les 2 seuls descendants du croisement A par $\mathrm{Cv}$ n'apportent aucun renseignement.

\section{Evaluation de la quantité moyenne de sporocystes formés par thalle à $16^{\circ} \mathrm{C}$}

La sporulation des souches parentales est un caractère stable lorsque les thalles ont une bonne croissance végétative. Un test $\mathrm{X}^{2}$ a permis de montrer qu'il en était de même pour tous les descendants: les 3 suspensions d'une même souche recueillies indépendamment appartiennent à une même population et les variations observées peuvent être attribuées au hasard. A partir de tous les comptages nous avons calculé une concentration moyenne de la population puis nous en avons déduit une quantité moyenne de sporocystes par thalle.

Pour faciliter l'interprétation des résultats, nous avons regroupé sur un même graphe, les valeurs obte-

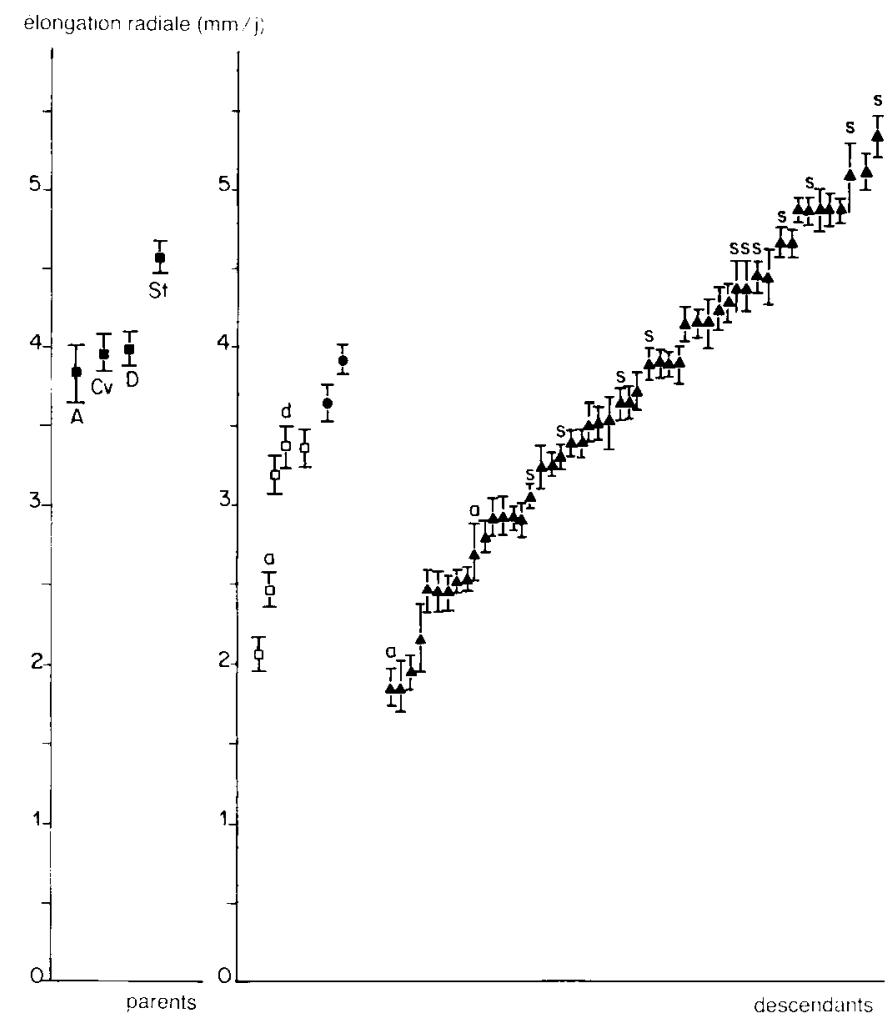

Figure 1

Elongation radiale moyenne par jour des souches parentales et de leurs descendants cultivés à $16^{\circ} \mathrm{C}$.

Daily radial growth for parental strains and their progeny.

- souches parentales;

$\square$ descendants de la confrontation $A$ par $D$;

- descendants de la confrontation $A$ par $C v$;

- descendants de la confrontation $A$ par $S t$;

a autofécondation de la souche $A$;

$\mathrm{d}$ autofécondation de la souche $D$;

$\mathrm{s}$ autofécondation de la souche St.

nues concernant les souches parentales et leurs descendants. Ces derniers sont classés en fonction de la quantité croissante de sporocystes formés par thalle (fig. 2).

Le parent $\mathrm{A}$, commun aux 3 séries de confrontations se distingue de ses différents partenaires en produisant peu de sporocystes, soit $7.10^{3}$ sporocystes par thalle contre $9.10^{6}$ pour $\mathrm{Cv}, 1,4.10^{7}$ pour St et $1,5.10^{7}$ pour D. Aucun des descendants ne sporule plus abondamment que le parent le plus performant, au mieux, il avoisine la production de celui-ci. En revanche 26 p. 100 d'entre eux, originaires des 3 types de crojsements différencient moins d'organes de propagation que le parent $\mathrm{A}$.

L'analyse de ce caractère, à travers la descendance du croisement A par St montre que la sporulation est aussi de nature quantitative en raison de la dispersion des valeurs observées.

\section{DISCUSSION}

L'étude de la croissance et de la sporulation à $16{ }^{\circ} \mathrm{C}$ à travers des descendances de première génération, et essentiellement celle du croisement A par St 


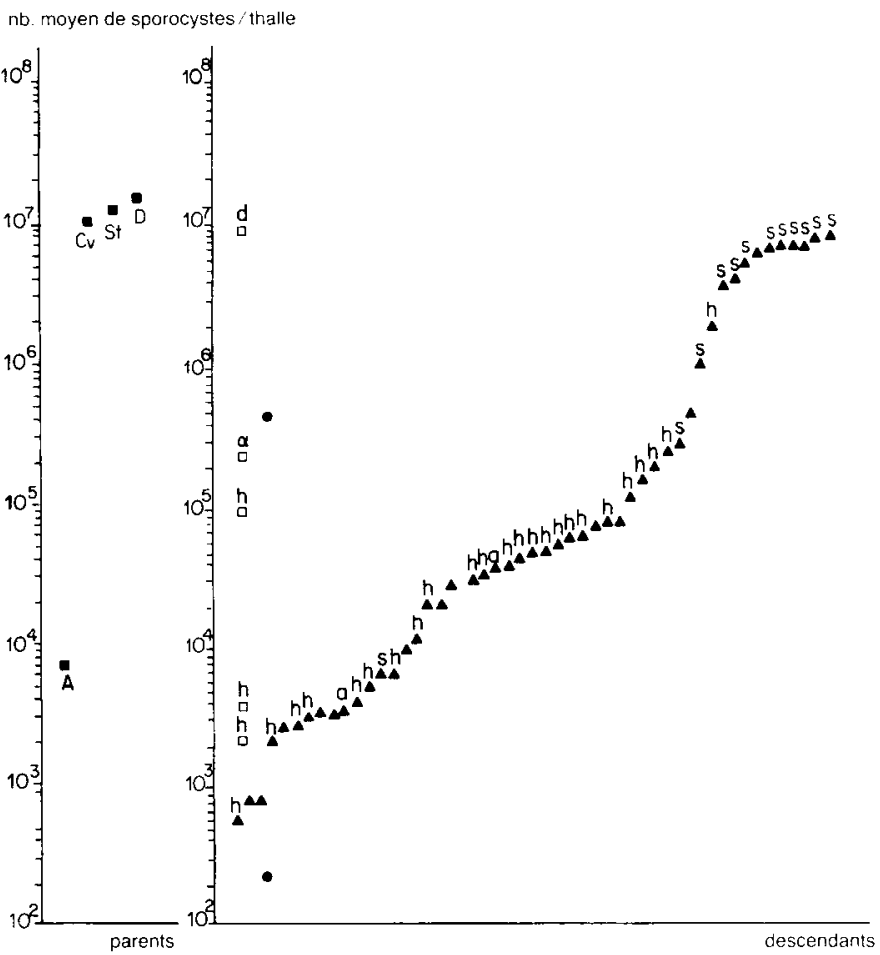

Figure 2

Evaluation de la quantité moyenne de sporocystes formés par thalle, pour les souches parentales $A, D$, St et $C v$ et les descendants issus des différentes confrontations.

Mean quantity of sporocysts per thallus for parental strains $A, D, S t$ and $C v$ and their progenies.

souches parentales;

$\square$ descendants de la confrontation $A$ par $D$;

- descendants de la confrontation $A$ par $C V$;

- descendants de la confrontation A par St;

a autofécondation de la souche $A$;

d autofécondation de la souche $D$;

s autofécondation de la souche St;

$\mathrm{h}$ hybrides. suggère que ces 2 caractères sont probablement de nature quantitative et qu'ils sont sous le contrôle de plusieurs gènes.

Lorsque l'on considère la figure 2 , on peut penser à première vue que cette courbe présente 3 paliers, dont les 2 plateaux extrêmes correspondraient approximativement aux valeurs parentales, et le plateau intermédiaire aux hybrides de première génération. On se placerait alors dans le cas d'une structure génétique homogène et différente chez chacun des parents, pour les gènes contrôlant ce caractère. En effet, dans la mesure où les autofécondations des 2 types se mêlent aux hybridations, comme l'étude du comportement à $10^{\circ} \mathrm{C}$ l'a montré, le palier inférieur regrouperait les autofécondés de la souche $\mathrm{A}$, le palier supérieur ceux de la souche $\mathrm{St}$, enfin la population homogène hybride déterminerait le palier central. Mais d'une part ce serait négliger toutes les autres valeurs qui ne se rattachent pas aux cas précédents et qui diffèrent entre elles, témoignant d'une ségrégation continue de la sporulation ; d'autre part ce serait ignorer pour certains descendants leur origine autofécondée ou hybride pressentie lors de l'étude du comportement à $10^{\circ} \mathrm{C}$ et généralement confirmée ou précisée lors de la détermination du signe de compatibilité sexuelle. Ainsi sur la figure 2, nous avons noté par a, h ou s, l'origine probable des descendants. Si une majorité de souches dont l'origine est soit l'autofécondation du parent St, soit l'hybridation entre $\mathrm{A}$ et $\mathrm{St}$, se situe respectivement au $3^{\mathrm{e}}$ et $2^{\mathrm{e}}$ niveau de la courbe, appuyant ainsi l'hypothèse de structures parentales homozygotes, la présence de nombreux hybrides, répartis tout le long de la courbe, ne peut être expliquée simplement que par l'existence dès l'origine d'hétéroallèles.

De plus cette nouvelle hypothèse permet de rendre compte de la grande diversité des phénotypes rencontrés lors de l'étude de la croissance. Ainsi la continuité de la dispersion des valeurs observées en première

TABLEAU 3

Regroupement des caractères étudiés chez les souches parentales $A$ et St et certains de leurs hybrides. Grouping of studied characters with parental strains and some of their hybrids.

\begin{tabular}{|c|c|c|c|c|c|}
\hline \multirow[b]{2}{*}{ Souches } & \multirow[b]{2}{*}{ Type sexuel } & \multicolumn{2}{|c|}{ Comportement à $10^{\circ} \mathrm{C}$} & \multicolumn{2}{|c|}{ Comportement à $16^{\circ} \mathrm{C}$} \\
\hline & & $\begin{array}{c}\text { Croissance } \\
(\%)\end{array}$ & Morphotype & $\begin{array}{l}\text { Croissance } \\
(\mathrm{mm} / \mathrm{j})\end{array}$ & $\begin{array}{l}\text { Sporulation } \\
\text { spores/thalles* }\end{array}$ \\
\hline A & A2 & 0 & M2 (a) & $3,84 \pm 0,20$ & $7,2 \quad 10^{3}$ \\
\hline $\mathrm{St}$ & $\mathrm{A} 1$ & 100 & M1 (b) & $4,51 \pm 0,11$ & $2000 A^{*}$ \\
\hline 21 & $\mathrm{~A} 1 / / \mathrm{A} 2$ & 20 & $\equiv \mathrm{St}$ & $\leq \mathrm{A}$ & $3 \mathrm{~A}^{*}$ \\
\hline 22 & $\mathrm{~A} 1 / / \mathrm{A} 2$ & 20 & $\equiv \mathrm{St}$ & $\equiv \mathrm{St}$ & $0,3 \mathrm{~A}$ \\
\hline 52 & $\mathrm{~A} 1 / / \mathrm{A} 2$ & 52 & $\simeq \mathrm{St}$ & $<\mathrm{A}$ & $8 \mathrm{~A}$ \\
\hline $\begin{array}{l}31 \\
18\end{array}$ & $\begin{array}{l}\text { Al } \\
\text { Al }\end{array}$ & $\begin{array}{l}52 \\
16\end{array}$ & \}$\neq \mathrm{A} \neq \mathrm{St}$ & $\begin{aligned} & <\mathrm{A} \\
\mathrm{A} & <\mathrm{w} 18<\mathrm{St}\end{aligned}$ & $\begin{array}{l}0,5 \mathrm{~A} \\
88 \mathrm{~A}\end{array}$ \\
\hline $\begin{array}{l}14 \\
49 \\
58\end{array}$ & $\begin{array}{l}\mathrm{A} 1 / / \mathrm{A} 2 \\
\mathrm{~A} 1 / / \mathrm{A} 2 \\
\mathrm{~A} 2\end{array}$ & & $\simeq \mathrm{St}$ & $\begin{array}{l}\equiv \mathrm{A} \\
<\mathrm{A}\end{array}$ & $\begin{array}{ll}0,8 & \mathrm{~A} \\
4,6 & \mathrm{~A} \\
4,3 & \mathrm{~A}\end{array}$ \\
\hline $\begin{array}{l}41 \\
50 \\
59 \\
61\end{array}$ & $\begin{array}{c}\mathrm{A} 2 \\
\mathrm{~A} 2 \\
\mathrm{~A} 1 / / \mathrm{A} 2 \\
\mathrm{~A} 2\end{array}$ & $\equiv$ St 100 & $\simeq \mathrm{St}$ & $\begin{aligned} \mathrm{A} & <\mathrm{w} 41<\mathrm{St} \\
& <\mathrm{A} \\
& \equiv \mathrm{A} \\
& <\mathrm{A}\end{aligned}$ & $\begin{array}{ll}3,3 & \mathrm{~A} \\
0,1 & \mathrm{~A} \\
0,7 & \mathrm{~A} \\
13 & \mathrm{~A}\end{array}$ \\
\hline $\begin{array}{l}15 \\
35 \\
60\end{array}$ & $\begin{array}{l}\mathrm{A} 1 \\
\mathrm{~A} 2 \\
\mathrm{~A} 1\end{array}$ & & $\neq \mathrm{A} \neq \mathrm{St}$ & $\begin{array}{l}>\mathrm{St} \\
\equiv \mathrm{St} \\
<\mathrm{A}\end{array}$ & $\begin{array}{l}9 \mathrm{~A} \\
0,5 \mathrm{~A} \\
\mathrm{~A}\end{array}$ \\
\hline
\end{tabular}

$\left({ }^{*}\right)$ Pour la souche St et les descendants nous avons tout rapporté au nombre moyen de spores produites par un thalle A. 
génération lors de cette étude (fig. 1), qui laisse penser qu'une multitude d'étapes phénotypiques peu différentes de l'une à l'autre doit correspondre au brassage d'un grand nombre de loci, ne peut s'expliquer que par l'hétérozygotie d'au moins 1 des 2 parents. Si l'on étudie la répartition des descendants autofécondés de la souche St marqués par un « $\mathrm{s}$ » sur la figure 1, le parent St serait bon candidat. Mais le croisement entre deux hétérozygotes n'est pas exclu comme semblent en témoigner les 3 descendants nés de l'autofécondation de A (fig. 1 et 2). Récemment, par une approche biochimique TOOLEY et FRY (1983) ont pu confirmer que la plupart des isolats de $P$. infestans testés étaient hétérozygotes pour de nombreux gênes.

Pour appuyer nos remarques nous avons regroupé dans le tableau 3 l'ensemble des caractères étudiés chez les 2 souches parentales $\mathrm{A}$ et $\mathrm{St}$ et quelques-uns de leurs descendants dont l'origine hybride est fortement soupçonnée. Ainsi nous considérons (tabl. 2A) les 3 ambivalents intermédiaires de morphotype M1, les 2 IM3, les 7 descendants, ambivalents ou de type A2, de morphotype St/M1a, St/M1c, dont l'origine ne peut pas être l'autofécondation de $A$, puisqu'ils ont une croissance totale à $10^{\circ} \mathrm{C}$, ni celle de St puisqu'ils sont de type $\mathrm{A}_{1} / / \mathrm{A}_{2}$ ou $\mathrm{A}_{2}$. Enfin les 3 descendants (St/M3) dont le morphotype à $10^{\circ} \mathrm{C}$ n'est ni celui de $\mathrm{A}$ ni celui de St et dont le signe est $\mathrm{A}_{1}$ ou $\mathrm{A}_{2}$.
Tous les hybrides considérés sont très différents, seul le caractère de sporulation à $16{ }^{\circ} \mathrm{C}$ semble, mis à part pour w18, relativement proche de celui de la souche $\mathrm{A}$ et très différent de la souche St. Toutefois, gardonsnous de généraliser ce cas à tous les descendants hybrides puisque ceux de type St/M2b (tabl. 2A) comme w10, w24, w55, w27 sporulent de 30 à près de 400 fois plus que la souche $A$.

L'hétérozygotie partielle que nous venons de soupçonner chez les souches parentales peut-elle s'étendre à d'autres caractères comme la virulence ? Les allèles de virulence correspondant à ceux des gènes de résistance spécifique chez l'hôte sont-ils récessifs ? S'il en est ainsi, hébergés dans une structure hétérozygote ils ne s'exprimeront pas. Seules la mutation ou les réassociations des déterminants raciaux par recombinaison sexuelle ou parasexuelle, conduisant à une homozygotie, pourraient, selon la pression de sélection exercée, permettre leur expression.

L'étude du comportement des descendants analysés ici, sur une gamme d'hôte différentielle, nous permettra d'apporter quelques éléments de réponse (LE GRAND-PERNOT, article soumis à Agronomie).

Reçu le 19 novembre 1985. Accepté le 8 août 1986.

\section{RÉFÉRENCES BIBLIOGRAPHIQUES}

Boccas B., 1971. Contribution à l'étude de la reproduction sexuelle chez Phytophthora palmivora, parasite des cultures tropicales. Thèse $3^{\mathrm{e}}$ Cycle, Orsay, $56 \mathrm{p}$.

Boccas B., Zentmyer G. A., 1976. Genetical studies with interspecific crosses between Phytophthora cinnamomi and Phytophthora parasitica. Phytopathology, 66, 477-484.

Boceas B., 1978. La reproduction sexuée chez les Phytophthora. Ses voies et quelques-unes de ses conséquences génétiques. Thèse Doct. Etat, Orsay, $175 \mathrm{p}$.

Dagnelie P., 1970. Théorie et méthodes statistiques. Applications agronomiques. Vol. II, Duculot, S.A. Gembloux, 445 p.

Gallegly M. E., 1970. Genetics of Phytophthora. Phytopathology, 60, 1135-1141

Gallegly M. E., Galindo J., 1958. Mating types and oospores of Phytophthora infestans in nature in Mexico. Phytopathology, 48, 274-277

Khaki J. A., Shaw D. S., 1974. The inheritance of drug resistance and compatibility type in Phytophthora drechsleri. Genet. Res. Camb., 23, 75-86.

Laviola C., 1974. Genetica di Phytophthora infestans (Mont.) de Bary. IV. Identificazione di un nuovo carattere genetico : produzione di sporangi. Phytopathol. medit., 13, 93-96.

Laviola C., Gallegly M. E., 1969. Segregation for physiologic race and compatibility type among F1 culture Phytophthora infestans. Phytopathology, 59, 1557-1558.

Le Grand-Pernot F., 1981. Facteurs influant sur le processus d'établissement de thalles monozoospores chez Phytophthora infestans. Can. J. Bot., 59, 836-841.

Le Grand-Pernot F., 1983. Tentative de domestication in vitro d'un champignon biotrophe Phytophthora infestans (Mont.) de Bary. Organisation du génome, ses conséquences. Thèse Doct. Etat, Orsay, $210 \mathrm{p}$.
Le Grand-Pernot F., Pellegrin F., 1976. Nature du cycle végétatif des Phytophthora parasitica Dastur et palmivora (Butler) Butler. Ann. Phytopathol., 8, 379-388.

Maia N., 1986. Contribution à l'étude de la transmission héréditaire de quelques caractères dans le genre Phytophthora. Thèse Doct. Etat, Orsay, 138 p.

Mortimer A. M., Shaw D. S., Sansome E. R., 1977. Genetical studies of secondary homothallism in Phytophthora drechsleri. Arch. Microbiol., 111, 255-259.

Paillard M., 1977. Nature du cycle végétatif de Phytophthora infestans. D.E.A. Amélioration des Plantes, Option Phytopathologie, $17 \mathrm{p}$.

Satour M. M., Butler E. E., 1968. Comparative morphological and physiological studies on the progenies from intraspecific mating of Phytophthora capsici. Phytopathology, 58, 183-192.

Savage J., Clayton C. W., Hunter J. H., Brenneman J. A., Laviola C., Gallegly M. E., 1968. Homothallism, heterothallism and interspecific hybridization in the genus Phytophthora. Phytopathology, 58, 1004-1021.

Shaw D. S., Khaki J. A., 1971. Genetical evidence for diploidy in Phytophthora. Genet. Res. Camb., 17, 165-167.

Shaw D. S., Janssen B., Khaki J. A., 1973. The genetics of Phytophthora drechsleri. Cah. ORSTOM Biol., 20, 57-58.

Timmer L. W., Castro J., Erwin D. C., Belser W. L., Zentmyer G. A., 1970. Genetic evidence for zygotic meioses in Phytophthora capsici. Amer. J. Bot., 57, 1211-1218.

Tooley P. W., Fry W. E., 1983. Genetic variation in Phytophthora infestans identified through isozyme analysis. Phytopathology, 73 (5), 827. 